\title{
A novel analytical approach to estimate isothermal and dynamic crystallization Avrami kinetic parameters
}

\author{
Hossein Ali Khonakdar, ${ }^{1 *}$ Masoud Shiri, ${ }^{1}$ Mahdi Golriz, ${ }^{1}$ Ahmad Asadinezhad, ${ }^{2}$ Seyed \\ Hassan Jafari, ${ }^{2}$ \\ $1^{*}$ Iran Polymer and Petrochemical Institute, P.O. Box: 14965-115, Tehran, Iran; fax: \\ +98-21-44580021; email: h.khonakdar@ippi.ac.ir, halikh@yahoo.com \\ ${ }^{2}$ School of Chemical Engineering, University of Tehran, P.O. Box: 11155-4563, \\ Tehran, Iran.
}

(Received: 13 November, 2007; published: 17 July, 2008)

\begin{abstract}
The Avrami equation has been extensively used to describe the polymer crystallization kinetics. Evaluation of kinetic parameters of this equation is quite troublesome, calling for properly predetermining induction time. To avoid this problem, an analytical approach in terms of differential scanning calorimetry exotherm maximum point was adopted here so as to conveniently estimate the Avrami equation kinetic parameters both in isothermal and dynamic crystallization mode. In the case of dynamic crystallization under isokinetic conditions, a modified version of the Avrami model by Nakamura was employed and adequate predictions based on the derived formulas were obtained. However, due to the simplified assumptions of the Avrami theory, our adopted relations are only applicable to earlier stages of crystallization process and do not account for secondary crystallization.
\end{abstract}

\section{Introduction}

Crystallization governs the macroscopic structure of the polymeric materials and thereby influences the performance of final products. Thus, it is vital to take into account the crystallization kinetics in the process modeling of crystallizable polymers $[1,2]$. Within the crystallization course, the crystals constitute multidimensional lamellar structures via the stages of nucleation and spherulite growth. As the temperature goes below the melting point, the molecules tend to move toward their lowest energy conformation, i.e., stiffer chains, and this favors the formation of ordered chains and then nuclei. Subsequent to the nucleation, crystals grow by the accumulation of the chains on the nuclei surfaces. This growth is dictated by a small diffusion coefficient at low temperature and by thermal redispersion of chains at the crystal/melt interface at high temperature [1, 3].

The analysis of overall crystallization provides invaluable information for modeling and control of polymer processing. It would be constructive to have models so fundamental in nature that one can insert basic characteristics for the material and gain realistic crystallization kinetics [4]. Phase transition-based analyses represent one of the most important topics in materials science since they are normally involved in the production cycle of materials. The kinetics of phase transitions plays a fundamental role in determination of the microstructure and therefore in mechanical properties of final product $[5,6]$. A well-known description of the crystallization process is the Avrami phase transition theory [7-9]. Though numerous models of 
crystal growth kinetics have been developed [4, 5], the Avrami equation grounded in rather empirical ideas is invoked not only to interpret polymer crystallization data but also to describe the curing behavior of resins $[10,11]$.

Determination of Avrami kinetic parameters is of crucial importance normally accomplished by fitting experimental data to the model. To avoid erroneous data, one has to follow a methodology on how the empirical data should be acquired and then analyzed on the basis of the model. Of significant sources of error, are induction time and the baseline of differential scanning calorimetry (DSC) crystallization exotherm, unless properly set, they may lead to substantial deviations from reality [12]. To overcome these problems, Lambrigger $[13,14]$ took advantage of an iterative procedure along with building crystallization-temperature independent master curves based on Avrami equation.

In this paper, we decide to adopt an analytical approach in order to facilitate estimating the values of kinetic parameters of the simplified Avrami theory, both in isothermal and dynamic mode, independent of the induction time and set of the baseline for DSC crystallization exotherm. This was accomplished by maintaining our focus on the DSC exotherm maximum point.

\section{Analysis}

\section{Isothermal Treatment}

To set the ground for the results described in this article, it seems indispensable to present a brief account of the most renowned phase transformation model by Avrami [7-9]. The Avrami equation which involves nucleation and growth concepts has manifested itself as a successful starting point for the analysis of polymer crystallization kinetics under isothermal conditions. The simplest mathematical form of the Avrami model assuming constant nucleation and linear growth rate reads as follows:

$$
X=\frac{X_{c}(t)}{X_{\infty}}=1-\exp \left(-Z t^{n}\right)
$$

where $X(t)$ is the relative crystallinity at time $t, X_{c}(t)$ is the absolute crystallinity at time $t, X_{\infty}$ is the ultimate absolute crystallinity, $Z$ is the isothermal crystallization rate constant containing contributions from nucleation and growth, and $n$ is the Avrami index. $Z$ and $n$ are specific to a given crystalline morphology and type of nucleation for a particular crystallization condition [15]. Further manipulations of Eq. (1) lead to the following expressions:

$$
\begin{aligned}
& -\ln (1-X)]=Z t^{n} \\
& \log [-\ln (1-X)]=\log Z+n \log t
\end{aligned}
$$

To determine the Avrami parameters ( $Z$ and $n$ ), the universally established method is to plot $\log [-\ln (1-X)]$ against $\log t$ and then estimate $Z$ and $n$. However, the values of $Z$ and $n$ to be calculated in this way are sensitive to one factor; induction (onset of crystallization) time, the time needed to obtain the first trace of crystallinity [4], whose determination is rather difficult. This can be realized well from Fig. 1 together with the following expressions in which reduced time is defined as $t^{*}=t-t_{o}$, where $t_{o}$ denotes 
induction time. Provided $t$ is replaced with $t^{*}$ in Eqs $1-3$, the following relation after some mathematical manipulations is deduced:

$$
n=\frac{\Delta \log [-\ln (1-X)]}{\Delta \log t^{*}}=\frac{\ln \left[\frac{-\ln \left(1-X_{2}\right)}{-\ln \left(1-X_{1}\right)}\right]}{\ln \left(\frac{t_{2}-t_{0}}{t_{1}-t_{0}}\right)}
$$

where $t_{1}$ and $t_{2}$ are two arbitrarily chosen times within the crystallization exotherm peak, and $X_{1}$ and $X_{2}$ are their corresponding relative crystallinity values. Note that $d H / d t$ in Fig. 1 signifies the crystallization enthalpy evolution rate. It is observed from Eq. (4) that the value of $n$ depends on the preset of induction time and if not properly determined, it can bring on an inaccurate $n$. To obtain the values of $n$ and $Z$ being independent of induction time, a calculus approach in terms of DSC isothermal crystallization peak is adopted which follows:

Starting from Eq. (2) in which $t$ is replaced with reduced time, we get to:

$[-\ln (1-X)]^{1 / n}=Z^{1 / n} t^{*}$

Provided Eq. (5) is set to $u$ and differentiated with respect to $t$, then:

$$
\frac{1}{n} \dot{u} u^{(1 / n)-1}=Z^{1 / n} \frac{d t^{*}}{d t}=Z^{1 / n}=\text { constant }
$$

in which $\dot{u}$ denotes the first derivative of $u$ with respect to time. Further differentiating Eq. (5) with respect to time results in:

$$
\ddot{u} u^{(1 / n)-1}+\left(\frac{1}{n}-1\right) \dot{u}^{2} u^{(1 / n)-2}=0
$$

where $\ddot{u}$ is the $2^{\text {nd }}$ derivative of $u$ with respect to time. If both sides of Eq. (7) are divided by nonzero expression of $u^{(1 / n)-1}$, then one reaches:

$\frac{1}{n}=1-\frac{\ddot{u} u}{\dot{u}^{2}}$

Since,

$u=-\ln (1-X) \Rightarrow \dot{u}=\frac{\dot{X}}{1-X} \Rightarrow \ddot{u}=\frac{\ddot{X}(1-X)+\dot{X}^{2}}{(1-X)^{2}}$

In consequence, Eq. (8) can be rewritten in this form:

$$
\frac{1}{n}=1+\frac{\left[\ddot{X}(1-X)+\dot{X}^{2}\right] \ln (1-X)}{\dot{X}^{2}}
$$

With particular regards to this notion that $\dot{X}$ and $\ddot{X}$ are equal to $\frac{-d H}{d t}$ and $\frac{-d^{2} H}{d t^{2}}$, respectively, thus, at the maximum point of DSC isothermal crystallization exotherm, the first derivative, $\dot{X}$, can be set to zero and therefore Eq. (10) may be simplified to the following useful relation: 


$$
\frac{1}{n}=1+\ln \left(1-X_{P}\right)
$$

where $X_{p}$ being the relative crystallinity at the exotherm peak. Based on Eq. (11), one is able to obtain the value of $n$ without having to preset $t_{0}$.

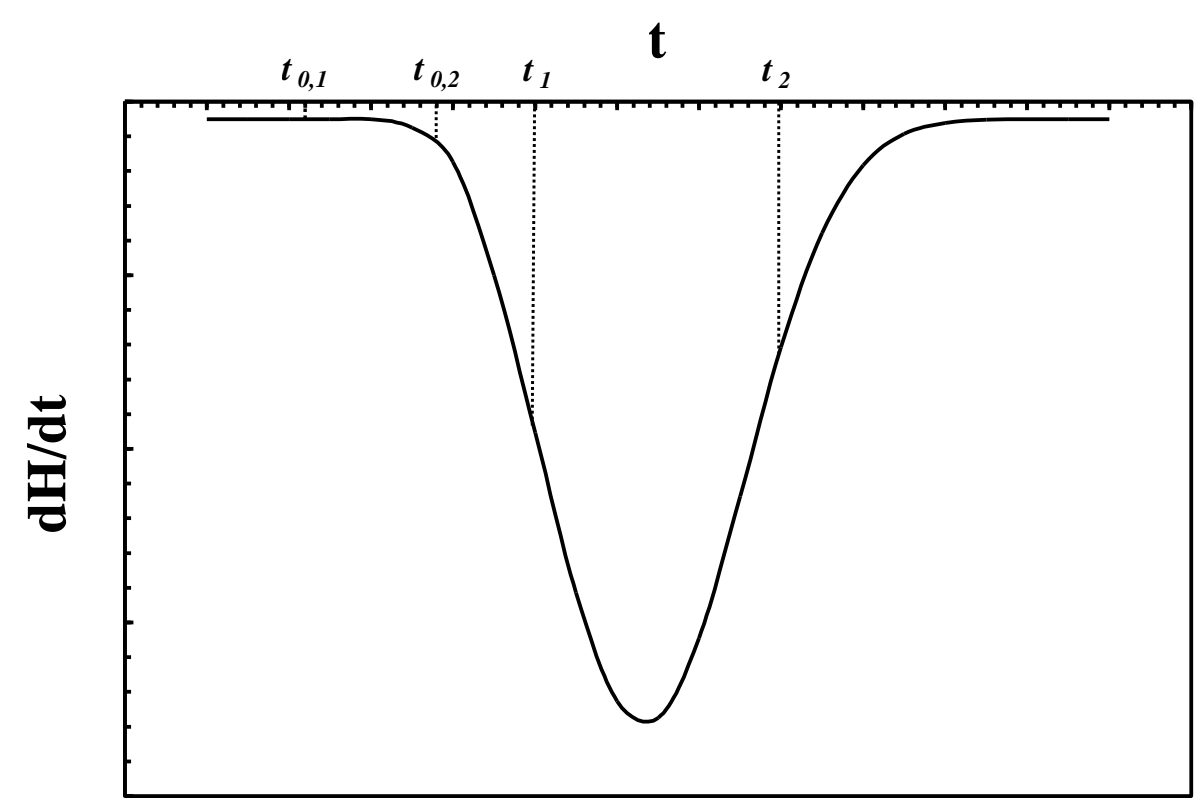

Fig. 1. A typical DSC exotherm peak obtained in an isothermal crystallization experiment. $t_{o, 1}$ and $t_{0,2}$ denote two induction times of choice.

An alternative approach to arrive at Eq. (11) is to differentiate Eq. (1) with respect to $t$ in which reduced time is substituted, as it follows:

$$
\begin{aligned}
& \dot{X}=\frac{d X}{d t}=n Z t^{*(n-1)} \exp \left(-Z t^{* n}\right) \\
& \ddot{X}=\frac{d \dot{X}}{d t}=n Z\left[(n-1) t^{*(n-2)}-n Z t^{*(2 n-2)}\right] \exp \left(-Z t^{* n}\right)
\end{aligned}
$$

Since at the maximum point of DSC exotherm $\ddot{X}$ is equal to zero, accordingly:

$$
Z t_{p}^{* n}=\frac{n-1}{n}=1-\frac{1}{n}
$$

By comparing Eqs (5) and (14), Eq. (11) is concluded.

The evaluation of Avrami rate constant, $Z$, and induction time, $t_{0}$, is also essential. In this regard, one can derive the following relations directly from Eq. (1) considering $t_{2}^{*}-t_{1}^{*}=t_{2}-t_{1}$ where $t_{1}$ and $t_{2}$ are two arbitrary instants chosen within the range of the onset of crystallization $\left(t_{o}\right)$ to exotherm maximum point $\left(t_{p}\right)$.

$$
Z=\left\{\frac{\left[-\ln \left(1-X_{2}\right)\right]^{1 / n}-\left[-\ln \left(1-X_{1}\right)\right]^{1 / n}}{t_{2}-t_{1}}\right\}^{n}
$$

As for induction time, from Eq. (5) one has: 
$t_{o}=t-\left[\frac{-\ln (1-X)}{Z}\right]^{1 / n}$

On the other hand, making use of Eqs (5) and (11), the following expression is concluded:

$$
Z^{1 / n} t_{p}^{*}=\mathbf{H} \ln \left(1-X_{P}\right)_{-}^{T / n}=\left(1-\frac{1}{n}\right)^{1 / n}
$$

$t_{p}$ is the moment when the exotherm reaches the maximum point. Finally, in light of Eqs (5), (11), and (17), one eventually obtains the useful simple relation shown below of which induction time may be estimated:

$$
t_{o}=t_{P}-\left(\frac{1-\frac{1}{n}}{Z}\right)^{1 / n}
$$

Piorkowska et al. [16] in an exhaustive review over the theories on the crystallization kinetics shed light on the fact that there may be significant discrepancies between the theoretical predictions of the classic Avrami model and experimental data arising from oversimplified assumptions. In other words, the crystallization process is by far more complicated than that described by Avrami theory. Thus, enough care should be taken while using such simplified relations to analyze the crystallization process in particular when Avrami index, due to some reason, becomes time dependent. In this case, the above equations are no longer valid.

\section{Non-isothermal Treatment}

The behavior of thermoplastic semicrystalline polymers over the non-isothermal crystallization course has drawn technological importance since it is the closest to the industrial processing conditions. Furthermore, from academic standpoint, a detailed survey of crystallization in dynamic conditions develops general insight into the crystallization behavior of polymers $[17,18]$.

Study on the dynamic crystallization kinetics from DSC has been approached in a theoretical way by Nakamura et al. [19]. Their model is an extension of the Avrami theory generalized to dynamic crystallization which allows the kind of nucleation and growth geometry to be predicted by factors analogous to Avrami parameters. Nakamura et al. [19] proposed that:

$$
X(t)=1-\exp \left[-\int_{0}^{t} K(T(\tau)) d \tau^{n}\right]
$$

which can be rearranged to :

$$
-\ln (1-X(t))=\int_{0}^{t} K(T(\tau)) d \tau^{n}
$$

where $K(T(\tau))$ is non-isothermal crystallization rate constant which is equal to $[Z(T)]^{1 / n}$, and $n$ and $Z$ are the Avrami index and isothermal crystallization rate constant respectively. $T$ is absolute temperature at time $\tau\left(\tau=t-t_{0}\right)$, and $Z$ has a dependency 
on temperature. Upon replacing $K(T(\tau))$ with $[Z(T)]^{1 / n}$ and inserting $t_{o}$ as the lower limit of integration, the Nakamura equation becomes:

$-\ln (1-X)=\left\{\int_{0}[Z(T(\tau))]^{1 / n} d \tau\right\}^{n}$

in which Avrami rate factor, $Z$, is inside the integral due to it's dependency on temperature.

Setting both sides of above relation to $u$ and then differentiating it with respect to time yields:

$\frac{1}{n} u^{(1 / n)-1} \dot{u}=[Z(T(\tau))]^{1 / n} \Rightarrow \frac{\dot{u}}{u}=n\left(\frac{Z(T)}{u}\right)^{1 / n}$

which can also be rewritten in the following form:

$$
\ln \left(\frac{\dot{u}}{u}\right)=\frac{1}{n} \ln \left(\frac{Z}{u}\right)+\ln (n)
$$

The following expression is obtained provided that Eq. (23) is differentiated in terms of time:

$$
\frac{1}{n}=\frac{\frac{\ddot{u}}{\dot{u}}-\frac{\dot{u}}{\dot{Z}}}{\frac{\dot{u}}{Z}-\frac{\dot{u} u}{u}}=\frac{\frac{\ddot{u}}{\dot{u}^{2}}-1}{\frac{\dot{Z} u}{Z \dot{u}}-1}
$$

In the vicinity of the maximum point of DSC exotherm peak, the fraction $\dot{Z} / Z$ is proportional to $\dot{T} / T$ with a proportionality factor close to one. On the other hand in this region $\dot{u} / u$ is sufficiently higher than the fraction $\dot{T} / T$ in such a way that the latter term can be neglected. This claim has been verified based on the empirical data reported for poly(trimethylene terephthalate) and polyamide-12 obtained at the cooling rate of $10 \mathrm{~K} / \mathrm{min}[20,21]$ as indicated by Fig. 2. It is also noteworthy that for $\dot{u} / u$ being 20 times as much as $\dot{T} / T$ close to the DSC peak, the error in assessment of Avrami index will be of the order of $5 \%$. The fraction $\dot{u} / u$ is independent of temperature variation rate, and depends only on the material, accordingly, the maximum temperature changes rate for which the above approximation holds is a function of $\dot{u} / u$.

In light of this approximation, Eq. (24) is reduced to:

$$
\frac{1}{n} \approx 1-\frac{\ddot{u} u}{\dot{u}^{2}}
$$

This relation is similar to what was obtained in previous section while dealing with isothermal conditions whose simplified form appears as:

$$
\frac{1}{n} \approx 1+\ln \left(1-X_{P}\right)
$$

Similar to what is declared at the end of the previous part, with special regards to the basic assumptions of the isokinetic model of Nakamura, one should note that such a simplified relation cannot give reliable data over a wide range of temperatures where 
isokinetic conditions do not sustain [16]. A very similar approach, however to the Ozawa model [22], was taken by Cazé et al. [17] and satisfactory results with respect to experimental data were reported.

It is noteworthy that for the purpose of accurately determining the Avrami kinetic parameters, we have prepared a software package based on differential equations derived from the Avrami and Nakamura models. This package is available for distribution upon request.
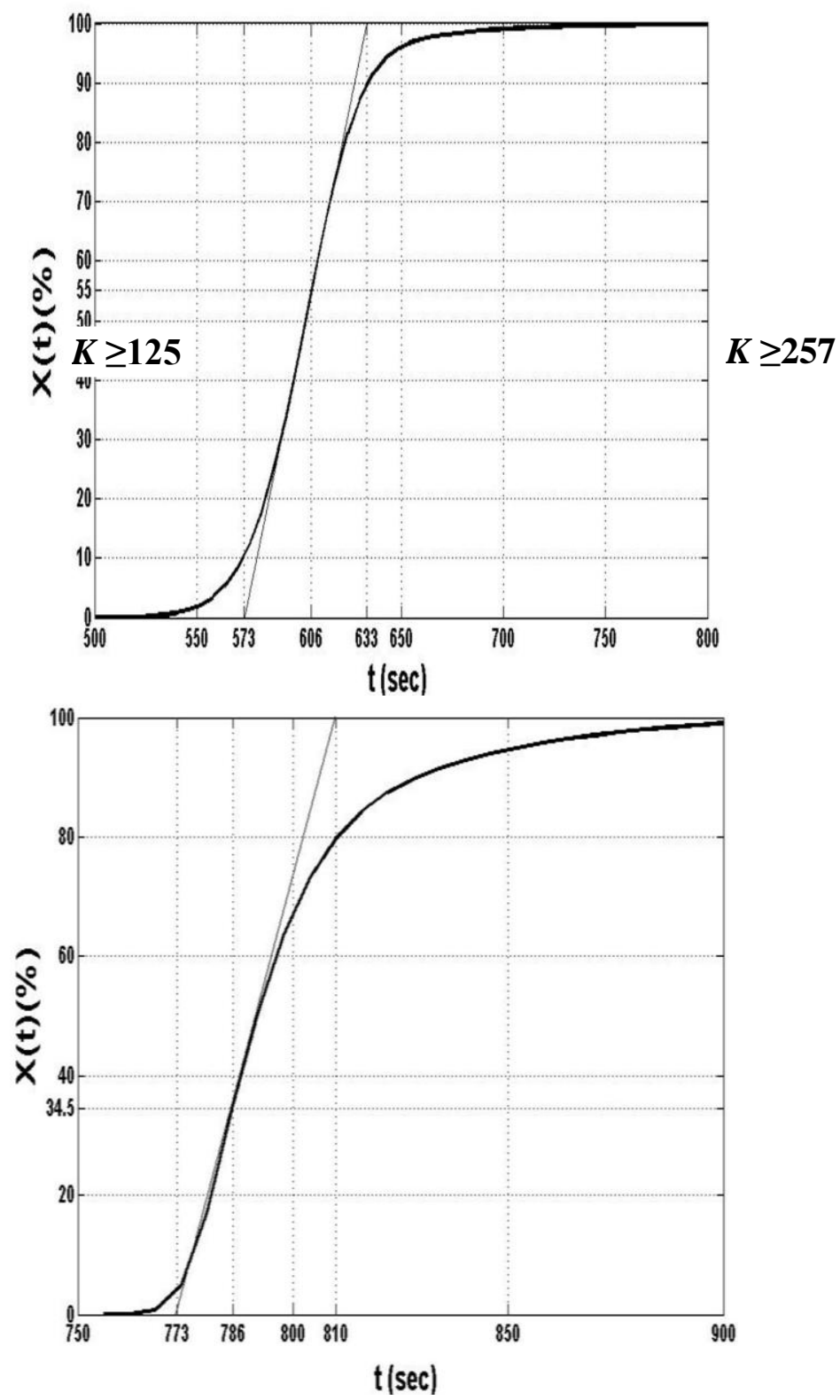

Fig. 2. Relative crystallinity vs. time for (a) poly (trimethylene terephthalate) and (b) polyamide-12 in which $\dot{u} / u$ is observed to be much higher than $\dot{T} / T$ - $K$ denotes the ratio of $\dot{u} / u$ to $\dot{T} / T$ (data from Refs. [20, 21]).

Fit with Experimental Data

The expressions derived in this contribution yield values for Avrami exponent being in accordance with the literature data. In a detailed work [23], Wang et al. analyzed the 
isothermal and dynamic crystallization of neat poly(trimethylene terephthalate) by means of Avrami equation and evaluated the Avrami exponents in different isothermal crystallization temperatures ( $T_{\text {iso }}$ (Fig. 3) making use of the classic method of curve fitting.

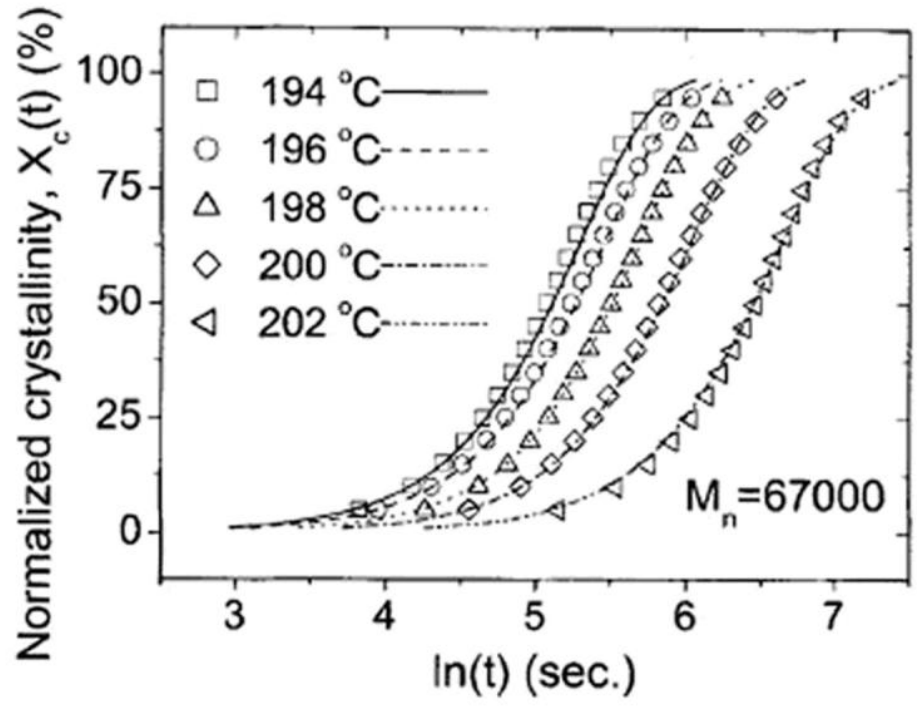

Fig. 3. Relative crystallinity vs. time for isothermal crystallization of poly(trimethylene terephthalate) at different crystallization temperatures (Ref. [23])

Tab. 1. The values of Avrami kinetic parameters evaluated at various crystallization temperatures by Eq. (11) compared to those obtained via classic procedure of curve fitting (Ref. [23])

\begin{tabular}{ccccc}
\hline $\begin{array}{c}T_{\text {iso }} \\
\left.\rho^{\mathrm{C}} \mathrm{C}\right)\end{array}$ & $n^{*}$ & $n^{* *}$ & $Z^{*}\left(\mathrm{~s}^{-n}\right)$ & $Z^{* *}\left(\mathrm{~s}^{-n}\right)$ \\
\hline 194 & 2.0 & 2.18 & $2.8 \times 10^{-5}$ & $1.8 \times 10^{-5}$ \\
196 & 2.0 & 2.16 & $2.2 \times 10^{-5}$ & $1.2 \times 10^{-5}$ \\
198 & 2.0 & 2.14 & $9.4 \times 10^{-6}$ & $5.4 \times 10^{-6}$ \\
200 & 2.0 & 2.12 & $6.1 \times 10^{-6}$ & $3.1 \times 10^{-6}$ \\
202 & 1.9 & 2.04 & $2.6 \times 10^{-6}$ & $1.6 \times 10^{-6}$ \\
\hline
\end{tabular}

Evaluated using curve fitting procedure

Evaluated using Eq. (11) and (15)

Tab. 1 gives a comparison of the Avrami exponent values calculated via Eq. (11) and those obtained from direct fitting to the Avrami model reported by Wang et al. [23]. According to the Tab. 1, a good agreement is observed among sets of data, although apparent discrepancy among $Z$ values pertains to strong dependency of $Z$ on the Avrami index ( $n$ ) which can be well understood from Eq. (15).

\section{Conclusions}

The Avrami equation has been extensively used to explain the polymer crystallization kinetics. The evaluation of kinetic parameters of Avrami equation and its modified form (Nakamura isokinetic model) generalized to dynamic crystallization is often a drudgery. The proposed approach in this article yields an effective transformation of the classical equations in order for the parameters to be directly linked with the 
characteristic points of DSC thermograms. With regards to fundamental assumptions of the Avrami theory, our derived formulas are only valid for primary crystallization and do not take into account secondary crystallization.

\section{References}

[1] Yang, J.; McCoy, B.J.; Madras, G. J. Chem. Phys. 2005, 122, 64901.

[2] Chen, C.M.; Higg, P.G. J. Chem. Phys. 1998, 108, 4305.

[3] Doye, J.K.; Frenkel, D. J. Chem. Phys. 1998, 109, 10033.

[4] Schultz, J.M. Polymer Crystallization, American Chemical Society, Washington, D.C. 2001.

[5] Long, Y.; Shanks, R.A.; Stachurski, Z.H. Prog. Polym. Sci. 1995, $20,651$.

[6] Sessa, V.; Fanfoni, M.; Tomellini, M. Phys. Rev. 1996, B54, 836.

[7] Avrami, M. J. Chem. Phys. 1939, 7, 1103.

[8] Avrami, M. J. Chem. Phys. 1940, 8, 212.

[9] Avrami, M. J. Chem. Phys. 1941, 9, 177.

[10] Lu, M.G.; Shim, M.J.; Kim, S.W. Thermochim. Acta 1998, 323, 37.

[11] Lu, M.G.; Shim, M.J.; Kim, S.W. J. Therm. Anal. Cal. 1999, 58,701.

[12] Lorenzo, A.T.; Arnal, M.L.; Albuerne, J.; Müller, A.J. Polym. Test. 2007, 26, 222.

[13] Lambrigger, M. Polym. Eng. Sci. 1996, 36, 610.

[14] Lambrigger, M. Polym. Eng. Sci. 1997, 37, 610.

[15] Wunderlich, B. Macromolecular Physics, Vol. 2, Academic Press, New York 1976.

[16] Piorkowska, E.; Galeski, A.; Haudin, J.M. Prog. Polym. Sci., 2006, 31, 549.

[17] Cazé, C.; Devaux, E.; Crespy, A.; Cavrot, J.P. Polymer 1997, 38, 497.

[18] Chan, T.W.; Isayev, A.I. Polym. Eng. Sci. 1994, 34, 461.

[19] Nakamura, K.; Katayama, K.; Amano, T. J. Appl. Polym. Sci. 1973, 17, 1031.

[20] Asadinezhad, A.; Jafari, S.H.; Khonakdar, H.A.; Böhme, F.; Hässler, R. Polymer Bulletin 2005, 54, 205.

[21] Khonakdar, H.A.; Jafari, S.H.; Asadinezhad, A. Iranian Polym. J.; 2008,17, 19.

[22] Ozawa, T. Polymer 1971, 12, 150.

[23] Wang, X.S.; Yan, D.; Tian, G.H.; Li, X.G. Polym. Eng. Sci. 2001, 41, 1655. 